\title{
Moderate calorie restriction attenuates age-associated alterations and improves cardiac function by increasing SIRT1 and SIRT3 expression
}

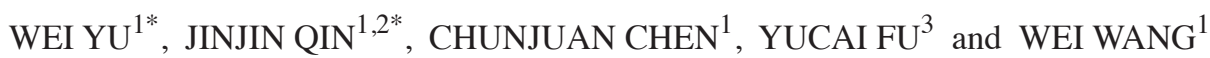 \\ ${ }^{1}$ Department of Cardiology, The Second Affiliated Hospital of Shantou University Medical College, Shantou, Guangdong 515041; \\ ${ }^{2}$ Department of Internal Medicine, Baoan Chronic Diseases Prevent and Cure Hospital, Shenzhen, Guangdong 518126; \\ ${ }^{3}$ Laboratory of Cell Senescence, Shantou University Medical College, Shantou, Guangdong 515041, P.R. China
}

Received June 16, 2017; Accepted November 16, 2017

DOI: $10.3892 / \mathrm{mmr} .2018 .9390$

\begin{abstract}
Calorie restriction (CR) extends the lifespan of mammals and improves cardiac function by attenuation of age-associated alterations. Sirtuins (SIRT) are involved in these mechanisms, however, the extent to which CR affects cardiac function and sirtuin expression remains unknown. Therefore, the present study aimed to determine to what extent $\mathrm{CR}$ affects cardiac function and sirtuin expression. A total of 60 female Sprague-Dawley rats were randomly divided into four groups, including normal control (NC), $25 \%$ calorie restriction $(25 \% \mathrm{CR}), 45 \%$ calorie restriction $(45 \% \mathrm{CR})$ and high-fat diet (HF). The groups were maintained on these specific regimens for 2 months. CR rats were observed to have significantly lower body weight, heart weight, and left ventricle mass index compared with $\mathrm{NC}$ and HF rats. Visceral fat, triglyceride, and low density lipoprotein levels were significantly decreased in CR rats. Compared with the $25 \%$ CR group, the $45 \%$ CR group heart function decreased. The heart rate, left ventricular systolic pressure, $+\mathrm{d} p / \mathrm{d} t$ and $-\mathrm{d} p / \mathrm{d} t$ of the $45 \% \mathrm{CR}$ rats decreased, whereas the left ventricular end-diastolic pressure increased. To explore the molecular mechanism of CR on cardiac function, immunoblotting was used to detect the protein expression of SIRT1 and SIRT3. The $25 \%$ CR diet increased the expression of SIRT1 and SIRT3 in myocardium, whereas the $45 \% \mathrm{CR}$ and HF diets resulted in a decrease in SIRT1 and SIRT3 expression. Moderate calorie
\end{abstract}

Correspondence to: Dr Wei Yu or Professor Wei Wang, Department of Cardiology, The Second Affiliated Hospital of Shantou University Medical College, 69 Dong-Xia North Road, Shantou, Guangdong 515041, P.R. China

E-mail: yuwei10610048@126.com

E-mail: wangwei_sumc@126.com

${ }^{*}$ Contributed equally

Key words: calorie restriction, cardiovascular diseases, metabolism, sirtuin 1 , sirtuin 3 restriction $(25 \% \mathrm{CR})$ improves cardiac function by attenuation of age-associated alterations in rats. SIRT1 and SIRT3 are associated with these effects.

\section{Introduction}

Obesity has become a worldwide public health problem. It has previously been demonstrated that excessive energy intake and obesity are considered to be risk factors for cardiovascular diseases $(1,2)$. Calorie restriction (CR), an effective non-pharmacological intervention of reduced energy intake, increases the health-span and life-span of worms and yeast to rats and fish (3). Several studies have reported that CR delays or decreases the development of various age-associated diseases, including cardiovascular disease, diabetes, and various forms of cancer (4-6). CR is important in regulating cardiovascular diseases via activation of silent information regulator 2 (SIR2) family members.

SIR2 is an $\mathrm{NAD}^{+}$-dependent deacetlyase and is associated with life-span extension in CR. There are seven SIR2 orthologs in mammals, sirtuin (SIRT) 1-7. SIRT1 is the closest and most well-characterized homolog of SIR2. SIRT1 mediates cellular metabolism and exerts corresponding effects on gene expression via deacetylating proteins, including the forkhead transcription factors (FOXOs), myogenic differentiation 1, the p53 tumor suppressor, and peroxisome proliferator-activated receptor- $\gamma$ co-activator $1 \alpha(7,8)$. Previous studies have demonstrated that the SIR2 family exhibits an important regulatory role in the pathogenesis of cardiovascular disease (9-12). In the mouse 12.5 day embryo, SIRT1 is expressed only in myocardial cells, however in adult rat myocardium, SIRT1 is expressed in the nucleus and the cytoplasm (13). SIRT1 deletion in rats leads to heart abnormalities, including cardiac atrial septal defects, ventricular septal defects and valvular deficiencies (14).

In addition to SIRT1, SIRT3, a stress-responsive deacetylase, which is localized in mitochondria, exhibits a positive effect in cardiac tissues. SIRT3 protects cardiomyocytes in stress situations via deacetylating Ku70 (15). Studies reveal SIRT3 knockout rats result in accelerated signs of aging in the heart including cardiac hypertrophy (16). 
It has been demonstrated that CR leads to upregulated expression of SIRT in numerous organs, including rodent brain, heart, liver and in white adipose tissues $(17,18)$. However, the extent to which CR affects cardiac function and SIRT expression is largely unknown.

The present study investigated the impact of moderate and severe calorie restriction compared with a high-fat dietary regimen on morphology and function of the cardiovascular system of aged rats. Furthermore, the effects of 25 and $45 \%$ CR diets on myocardial SIRT1 and SIRT3 expression levels were characterized.

\section{Materials and methods}

Ethics statement. The experiments were approved by the Institutional Animal Care and Use Committee of Shantou University Medical College (Shantou, China), and were conducted according to the Guide for the Care and Use of Laboratory Animals (The Regulation of Experimental Animals in Guangdong Province).

Materials. Primary antibodies against SIRT1, SIRT3 and $\beta$-actin were purchased from Santa Cruz Biotechnology, Inc. (Dallas, TX, USA). SIRT1 rabbit polyclonal IgG, cat. no. sc-15404; SIRT3, rabbit polyclonal IgG, cat. no. sc-49743; $\beta$-actin mouse monoclonal IgG, cat. no. sc-47778. An enhanced chemiluminescence (ECL) western blot detection system was obtained from GE Healthcare (Chicago, IL, USA).

Animals and treatment. A total of 60 8-week-old female Sprague-Dawley rats, weighing 178.1 g, were purchased from the Animal Center, Shantou University Medical College. The rats were housed separately in a temperatureand humidity-controlled animal house at $22-25^{\circ} \mathrm{C}$ with a 12 h-12 h light-dark cycle. Following one week of acclimatization feeding, rats were randomly divided into four groups (15 rats each): a normal control group (NC) fed with standard rodent chow containing 4\% fat, $4.5 \%$ fiber, and $24 \%$ protein, ad libitum; a $25 \%$ calorie restriction group ( $25 \% \mathrm{CR})$, fed with $75 \%$ of the total caloric intake from the NC group; a $45 \%$ calorie restriction group ( $45 \% \mathrm{CR}$ ), fed with $55 \%$ of the total caloric intake from the NC group; a high-fat group (HF) fed with a high-fat diet containing $10 \%$ lard, $3 \%$ yolk, $1 \%$ cholesterol, $0.5 \%$ sodium cholate, and $85.5 \%$ standard rodent chow. Sufficient vitamins and minerals were present in each of the diets, and the rats had unlimited access to water for 8 weeks. Rats were monitored and weighed daily.

Cardiac haemodynamic measurements. A total of eight weeks following treatment, left ventricular (LV) performance was measured in rats anaesthetized with intraperitoneal injections of $10 \%$ chloral hydrate $(250 \mathrm{mg} / \mathrm{kg})$. After observation for $5 \mathrm{~min}$, if the anaesthetic effect was not satisfactory, another dose of $100 \mathrm{mg} / \mathrm{kg}$ was added. The rats were placed on controlled heating pads, and the core temperature, measured via a rectal probe, was maintained at $36-38^{\circ} \mathrm{C}$. According to a previously described method (19), a small cannula filled with heparin saline $(500 \mathrm{U} / \mathrm{ml})$ was inserted into the left ventricle through the apex with the chest open and mechanically ventilated, and positioned along the cardiac longitudinal axis. Following stabilization for $2 \mathrm{~min}$, the pressure signal was continuously recorded using a MacLab A/D converter (AD Instruments, Mountain View, CA, USA). The left ventricular systolic and end-diastolic pressures were measured, and the maximal slope of systolic pressure increment $(+\mathrm{d} P / \mathrm{d} t)$ and diastolic pressure decrement $(-\mathrm{d} P / \mathrm{d} t)$ were calculated. Following obtainment of the haemodynamic measurements, the rats were sacrificed.

Sample collection and tissue processing. Blood samples were collected from tail veins every two weeks following fasting for $16 \mathrm{~h}$. Sera were immediately isolated from blood samples by centrifugation at $1,200 \mathrm{x}$ g for $5 \mathrm{~min}$ at $4^{\circ} \mathrm{C}$ and stored at $-20^{\circ} \mathrm{C}$ until analyzed. Hearts were harvested and flushed with $0.9 \%$ normal saline solution, prior to being sectioned into three parts. The central sections of each rat heart, which included dual atriums and dual ventricles, were used for western blot analysis and were immediately stored at $-80^{\circ} \mathrm{C}$.

Western blot analysis. Protein was extracted from heart tissues using radioimmunoprecipitation assay buffer $(1 \%$ Triton-X-100, $150 \mathrm{mmol} / 1 \mathrm{NaCl}, 5 \mathrm{mmol} / \mathrm{l}$ EDTA and $10 \mathrm{mmol} / \mathrm{l}$ Tris-HCl, $\mathrm{pH}$ 7.0) containing a protease inhibitor cocktail (Sigma-Aldrich; Merck KGaA, Darmstadt, Germany). The tissue lysates were subjected to centrifugation at 13,200 g for $10 \mathrm{~min}$ at $4^{\circ} \mathrm{C}$. Protein concentration was determined using the Bradford method. A total of $50 \mu \mathrm{g}$ protein per lane was separated by $12 \%$ SDS-PAGE and transferred onto a polyvinylidene fluoride membrane (EMD Millipore, Billerica, MA, USA). Following three washes with PBS, the membranes were soaked in $5 \%$ non-fat dry milk for $2 \mathrm{~h}$ at room temperature and incubated with primary antibodies against $\beta$-actin (dilution, 1:1,000), SIRT1 (dilution, 1:200) and SIRT3 (dilution, 1:200) overnight at $4^{\circ} \mathrm{C}$. Membranes were then incubated with goat anti-rabbit (cat. no. sc-2004) and goat anti-mouse (cat. no. sc-2005) horseradish peroxidase-conjugated IgG antibodies (dilution, 1:2,000; Santa Cruz Biotechnology Inc., Dallas, TX, USA) for $1 \mathrm{~h}$ at room temperature. Immune complexes were subsequently visualized using ECL and the band intensities were measured and quantified using Quantity One ${ }^{\circledR}$ software (Bio-Rad Laboratories Inc., Hercules, CA, USA).

Statistical analysis. All data are presented as the mean \pm standard deviation of at least three independent experiments. Statistical analyses were performed using one-way analysis of variance followed by Student-Newman-Keuls post hoc test for the comparison of multiple groups. All data were analyzed using SPSS v. 19.0 (IBM Corp., Armonk, NY, USA). P<0.05 was considered to indicate a statistically significant difference.

\section{Results}

Effect of CR on the body weight (BW), heart weight $(H W)$ and left ventricle mass index (LVMI) in rats. The $25 \% \mathrm{CR}$ rats maintained their initial weight until the end of the study, and their body weights were decreased compared with $\mathrm{NC}$ and $\mathrm{HF}$ groups $(\mathrm{P}<0.05$; Fig. 1A), however their HW and LVMI were similar to the NC and HF groups (Fig. 1B and C). The $45 \%$ $\mathrm{CR}$ rats exhibited the lowest $\mathrm{BW}$ following the experiment $(\mathrm{P}<0.01$; Fig. 1A), and HW and LVMI were also the lowest $(\mathrm{P}<0.05$; Fig. $1 \mathrm{~B}$ and $\mathrm{C})$. There was no significant difference 

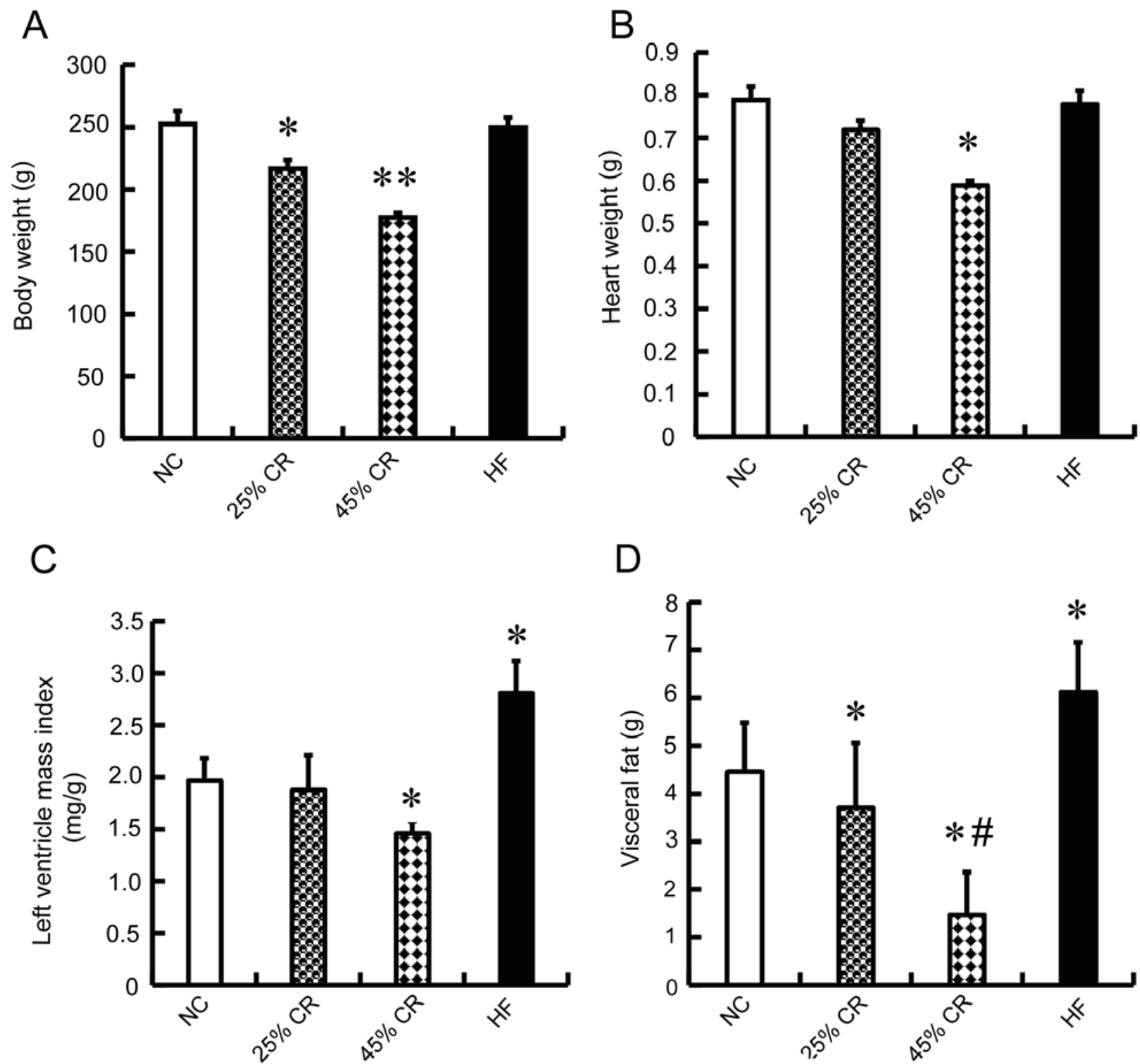

Figure 1. Effect of CR on the BW, HW, LVMI and VF in rats. The rats were randomized into four groups (15 mice each) with similar BW, a NC group, 25, 45\% CR and a HF group. (A) BW, (B) HW, (C) LVMI and (D) VF were significantly decreased with 45\% CR. There was no significant difference between HF and $\mathrm{NC}$ groups in BW and HW, however the LVMI and VF weight of the HF group was increased significantly compared with the other three groups. Data are presented as the mean \pm standard deviation. ${ }^{*} \mathrm{P}<0.05$ vs. the $\mathrm{NC}$ group; ${ }^{* *} \mathrm{P}<0.01$ vs. the $\mathrm{NC}$ group; ${ }^{*} \mathrm{P}<0.05$ vs. the $25 \% \mathrm{CR}$ group. BW, body weight; HW, heart weight; LVMI, left ventricle mass index; VF, visceral fat; $25 \% \mathrm{CR}$, a $25 \%$ calorie restriction group; $45 \% \mathrm{CR}, 45 \%$ calorie restriction group; HF, high-fat; NC, negative control.

between HF and NC groups in BW and HW (Fig. 1A and B), however the LVMI of the HF group was increased significantly compared with the other three groups $(\mathrm{P}<0.05$; Fig. 1C).

Effect of $C R$ on the visceral fat (VF), triglyceride (TG) and low density lipoprotein levels $(L D L)$ in rats. As presented in Fig. 1D, compared with the NC group, the two CR treated rats had decreased VF weight $(\mathrm{P}<0.05)$, whereas the VF weight was significantly increased in the HF group $(\mathrm{P}<0.05)$. The $45 \%$ CR group had the lowest, and there was a significant difference between the VF levels of the 25 and $45 \% \mathrm{CR}$ groups $(\mathrm{P}<0.05)$. TG levels in the HF-treated and $\mathrm{NC}$-treated groups increased rapidly in the first two weeks, decreased in the following weeks, however were increased at all time points compared with the CR groups $(\mathrm{P}<0.05)$. However, there was no significant difference between $\mathrm{HF}$ and $\mathrm{NC}$ groups. The TG levels of the 25 and $45 \%$ CR rats decreased $(P<0.05)$, however there was no significant difference between the CR groups (Table I). The LDL levels the in HF-treated rats was significantly increased at all time points $(\mathrm{P}<0.05)$. The LDL levels in other three groups demonstrated similar alterations, slightly increased, however there was no significant difference among the three groups (Table I).

Effect of CR on the LV function in rats. The heart rate is a good indicator of the cardiac function. The present study measured the heart rate and it was revealed that the $45 \%$ CR group rats' heart rate decreased, whereas the $25 \% \mathrm{CR}$ group increased $(\mathrm{P}<0.05$; Fig. 2A). Haemodynamic measurements were also recorded to determine the effect of $\mathrm{CR}$ on $\mathrm{LV}$ function in 
Table I. Effect of CR on the TG and LDL levels in rats.

\begin{tabular}{lllll}
\hline Variable & NC & $25 \% \mathrm{CR}$ & $45 \% \mathrm{CR}$ \\
\hline TG (mmol/l) & & & & \\
Week 2 & $0.78 \pm 0.11$ & $0.82 \pm 0.08$ & $0.88 \pm 0.05$ & $0.89 \pm 0.09$ \\
Week 4 & $1.09 \pm 0.15$ & $0.89 \pm 0.21^{\mathrm{a}, \mathrm{b}}$ & $0.84 \pm 0.13^{\mathrm{a}, \mathrm{b}}$ & $1.32 \pm 0.06^{\mathrm{a}}$ \\
Week 6 & $0.81 \pm 0.23$ & $0.57 \pm 0.18^{\mathrm{a}, \mathrm{b}}$ & $0.57 \pm 0.31^{\mathrm{a}, \mathrm{b}}$ & $0.96 \pm 0.19^{\mathrm{a}}$ \\
Week 8 & $0.86 \pm 0.17$ & $0.67 \pm 0.13^{\mathrm{a}, \mathrm{b}}$ & $0.55 \pm 0.13^{\mathrm{a}, \mathrm{b}}$ & \\
LDL (mmol/l) & & & $0.33 \pm 0.12$ & $0.12 \pm 0.19^{\mathrm{a}}$ \\
Week 2 & $0.34 \pm 0.10$ & $0.31 \pm 0.06$ & $0.32 \pm 0.12^{\mathrm{b}}$ & $0.41 \pm 0.15$ \\
Week 4 & $0.38 \pm 0.05$ & $0.31 \pm 0.11^{\mathrm{b}}$ & $0.43 \pm 0.20^{\mathrm{b}}$ & $0.53 \pm 0.14$ \\
Week 6 & $0.40 \pm 0.13^{\mathrm{b}}$ & $0.37 \pm 0.09^{\mathrm{b}}$ & $0.43 \pm 0.12^{\mathrm{b}}$ & $0.61 \pm 0.19$ \\
Week 8 & $0.46 \pm 0.05^{\mathrm{b}}$ & $0.44 \pm 0.05^{\mathrm{b}}$ & & \\
\hline
\end{tabular}

Data are expressed as the mean \pm standard deviation. The rats were randomized into four groups (15 mice each) with similar body weight, a normal control group (NC), a 25\% calorie restriction group (25\% CR), a 45\% calorie restriction group (45\% CR), a high-fat group (HF). LDL, low density lipoprotein; TG, triglyceride. ${ }^{\mathrm{a}} \mathrm{P}<0.05$ vs. the $\mathrm{NC}$ group; ${ }^{\text {b }} \mathrm{P}<0.05$ vs. the $\mathrm{HF}$ group.

rats. Compared with the NC group, the HF group rats exhibited a significant deterioration in haemodynamic indices in LVSP (96.4 \pm 6.46 vs. $112.8 \pm 7.08 \mathrm{mmHg}, \mathrm{P}<0.05$; Fig. 2B), LVEDP (7.6 \pm 0.81 vs. $6.8 \pm 0.53 \mathrm{mmHg}, \mathrm{P}<0.05$; Fig. $2 \mathrm{C}$ ), $+\mathrm{d} P / \mathrm{d} t(5,212 \pm 453$ vs. $6,030 \pm 486 \mathrm{mmHg} / \mathrm{s}, \mathrm{P}<0.05$; Fig. $2 \mathrm{D})$, and $-\mathrm{d} P / \mathrm{d} t(4,302 \pm 368$ vs. $5,120 \pm 467 \mathrm{mmHg} / \mathrm{s}, \mathrm{P}<0.05$; Fig. 2E). However, rats treated with $25 \%$ CR exhibited significant improvement in these haemodynamic indices $(\mathrm{P}<0.05$, Fig. 2B-E). Notably, the $45 \%$ CR group had a significant deterioration in haemodynamic indices compared with the $25 \%$ CR group in LVSP (81.7 \pm 9.56 vs. $131.5 \pm 7.14 \mathrm{mmHg}, \mathrm{P}<0.05$; Fig. 2B), LVEDP (7.9 \pm 0.55 vs. $6.0 \pm 1.02 \mathrm{mmHg}, \mathrm{P}<0.05$; Fig. $2 \mathrm{C}),+\mathrm{d} P / \mathrm{d} t(5,004 \pm 512$ vs. $6,860 \pm 566 \mathrm{mmHg} / \mathrm{s}, \mathrm{P}<0.05$; Fig. 2D), and $-\mathrm{d} P / \mathrm{d} t(4,198 \pm 499$ vs. $5,978 \pm 412 \mathrm{mmHg} / \mathrm{s}$, $\mathrm{P}<0.05$; Fig. 2E).

Western blot analysis of SIRT1 and SIRT3 protein expression. To explore the molecular mechanism of CR on cardiac function, immunoblotting was used to detect the protein expression of SIRT1 and SIRT3. The expression of SIRT1 and SIRT3 in the $25 \% \mathrm{CR}$ group was increased compared with the other three groups $(\mathrm{P}<0.05$, Fig. 3A-C). The $45 \% \mathrm{CR}$ and $\mathrm{HF}$ groups had decreased levels $(\mathrm{P}<0.05$, Fig. 3A-C) compared with NC group, and there was no difference between $45 \% \mathrm{CR}$ and $\mathrm{HF}$ groups.

\section{Discussion}

Obesity raises the risk of morbidity from hypertension, dyslipidemia, type 2 diabetes mellitus (diabetes), coronary heart disease, stroke, gallbladder disease, osteoarthritis, sleep apnea, respiratory problems and various cancers (20). Obesity is also associated with increased risk in all-cause and cardiovascular disease mortality (20). Although CR as a nonpharmacological intervention of reduced energy intake exhibits various benefits, including extending lifespan and delaying age-associated diseases (3-5), excessive CR may lead to a converse result.

In the present study, rats treated with a HF diet had the heavier BW, increased LDL level, greater TG level, and more abdominal fat. Conversely, rats treated with a $25 \% \mathrm{CR}$ diet and a $45 \%$ CR diet were much healthier with decreased BW, lower LDL levels, lower TG levels, and less abdominal fat. The $45 \%$ CR group demonstrated the greatest decrease. However, the rats of the $45 \%$ CR group exhibited various pathological features; their fur was sparser compared with the other three groups. Their daily activities were also not as energetic. The heart rate is a good indicator of cardiac function. In the appropriate range, the faster the heart rate, the better the cardiac function. At the end of the study, the heart rates were measured and it was demonstrated that the $45 \%$ CR group heart rates decreased, whereas the $25 \% \mathrm{CR}$ group heart rates increased. This indicated that excessive CR may be harmful to health.

It is well known that excessive energy intake may induce insulin resistance, central obesity, dyslipidemia and hypertension, which are all important risk factors of cardiovascular disease $(1,2,21)$. Dyslipidemia has been proven to have an important role in the development of cardiovascular diseases $(22,23)$. SIRT1 and SIRT3 may regulate lipid metabolism. SIRT1 is a positive regulator of liver $\mathrm{X}$ receptor proteins, nuclear receptors that function as cholesterol sensors and regulate whole body cholesterol and lipid homeostasis. SIRT1 knockout rats with a high-fat diet lead to high TG and high LDL levels (24). SIRT3 may activate mitochondrial fatty acid oxidation enzymes (12), suggesting SIRT3 may modulate lipid metabolism. All these studies may explain why HF rats accumulated fat mass and high blood fat whereas CR rats did not, although their body weights have no significant difference.

SIRTs demonstrate diverse cellular localizations and numerous cellular functions. The authors previously demonstrated that SIRT1 was identified in the nucleus and cytoplasm, whereas SIRT3 was predominantly observed in the cytoplasm (25). Moderate CR may upregulate SIRT expression in various organs, including cardiac tissues. It has previously been demonstrated that the mRNA and protein expression of SIRT1-4 and -7 are significantly increased in the cardiac tissues of rats in CR groups (25). However, it is still unclear whether excessive CR has any effect on SIRT expression. The results of the present study suggested that excessive CR resulted in a 

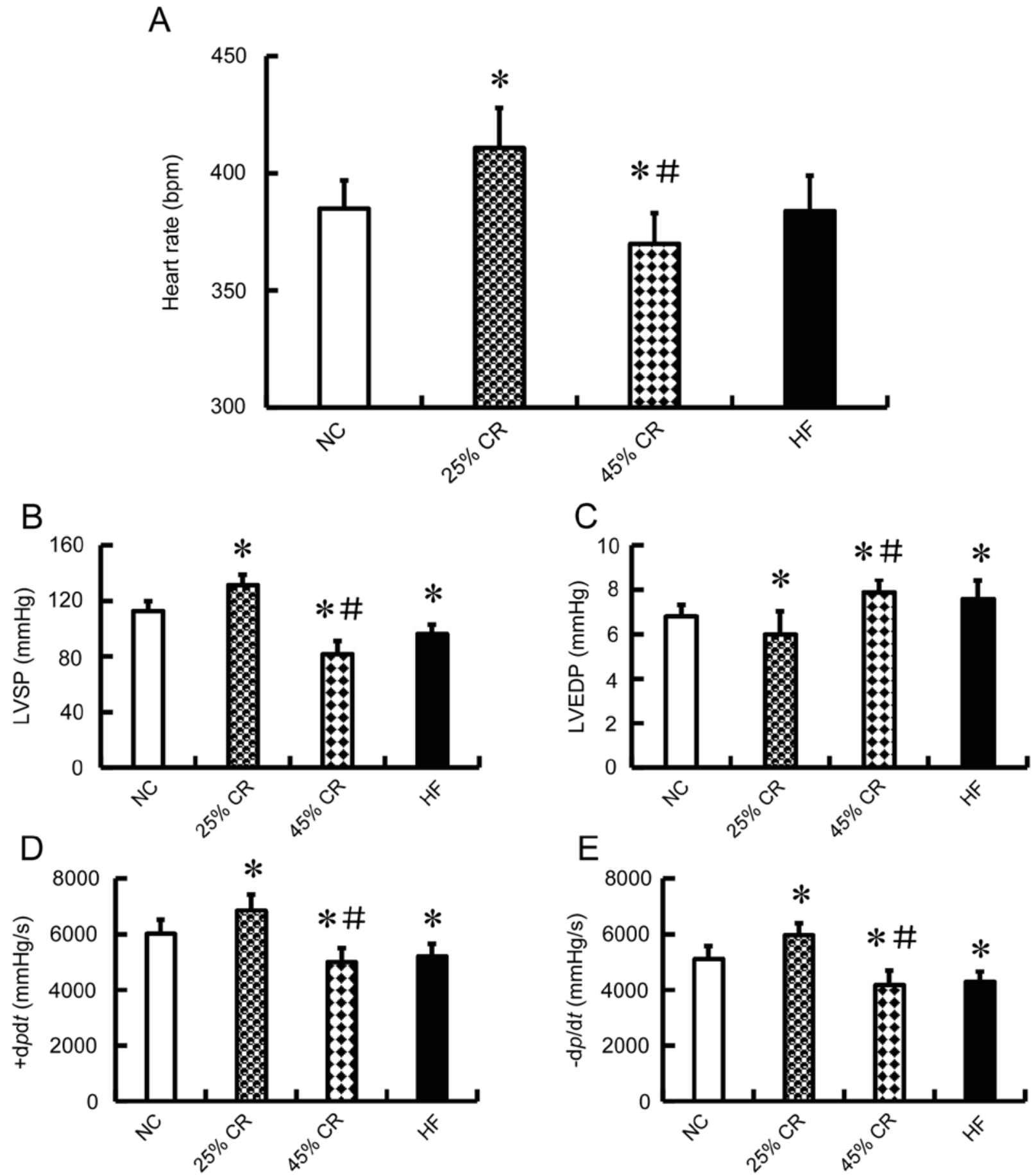

Figure 2. Effect of $\mathrm{CR}$ on the $\mathrm{LV}$ function in rats. The rats were randomized into four groups (15 mice each) with similar BW, a NC group, 25, 45\% CR and a HF group. A total of eight weeks following treatment, (A) HR was measured and it was demonstrated that the 45\% CR group HR decreased, whereas the 25\% CR group HR increased. Furthermore, LV performance was measured in rats anaesthetized by intraperitoneal injection of chloral hydrate. The haemodynamic variables between different treatment groups are presented. Alterations in (B) LVSP and (C) LVEDP. (D) The maximal slope of systolic pressure increment $(+\mathrm{d} P / \mathrm{d} t)$. (E) The maximal slope of diastolic pressure decrement $(-\mathrm{d} P / \mathrm{d} t)$. Data are presented as the mean \pm standard deviation. ${ }^{*} \mathrm{P}<0.05$ vs. the NC group; ${ }^{\#} \mathrm{P}<0.05$ vs. the $25 \% \mathrm{CR}$ group; $\mathrm{n}=8$. HR, heart rate; LVSP, left ventricular systolic pressure; LVEDP, left ventricular end-diastolic pressure; $25 \% \mathrm{CR}$, a $25 \%$ calorie restriction group; $45 \% \mathrm{CR}$, $45 \%$ calorie restriction group; HF, high-fat; NC, negative control; LV, left ventricular.

decline in SIRT expression. Expression levels of SIRT1 and SIRT3 in $45 \%$ CR were decreased compared with the $25 \%$ CR group, and were also decreased compared with NC.

Previous studies demonstrate that high expression of SIRT1 in transgenic rat hearts decreases cell apoptosis, cardiac dysfunction and cardiac hypertrophy $(26,27)$. Increasing expression of SIRT3 protects cardiac tissues from oxidative stress-mediated cell death (28). SIRT3-deficient mice at 8-weeks of age have a basal level of cardiac hypertrophy, and a concomitant decline in the protective effect of cardiac tissues (16). In the present study, the rats HW of the HF group was not significantly different compared with the $\mathrm{NC}$ and 25\% CR groups; however, the LVMI of the HF rats was significantly different compared with the other groups. 
A
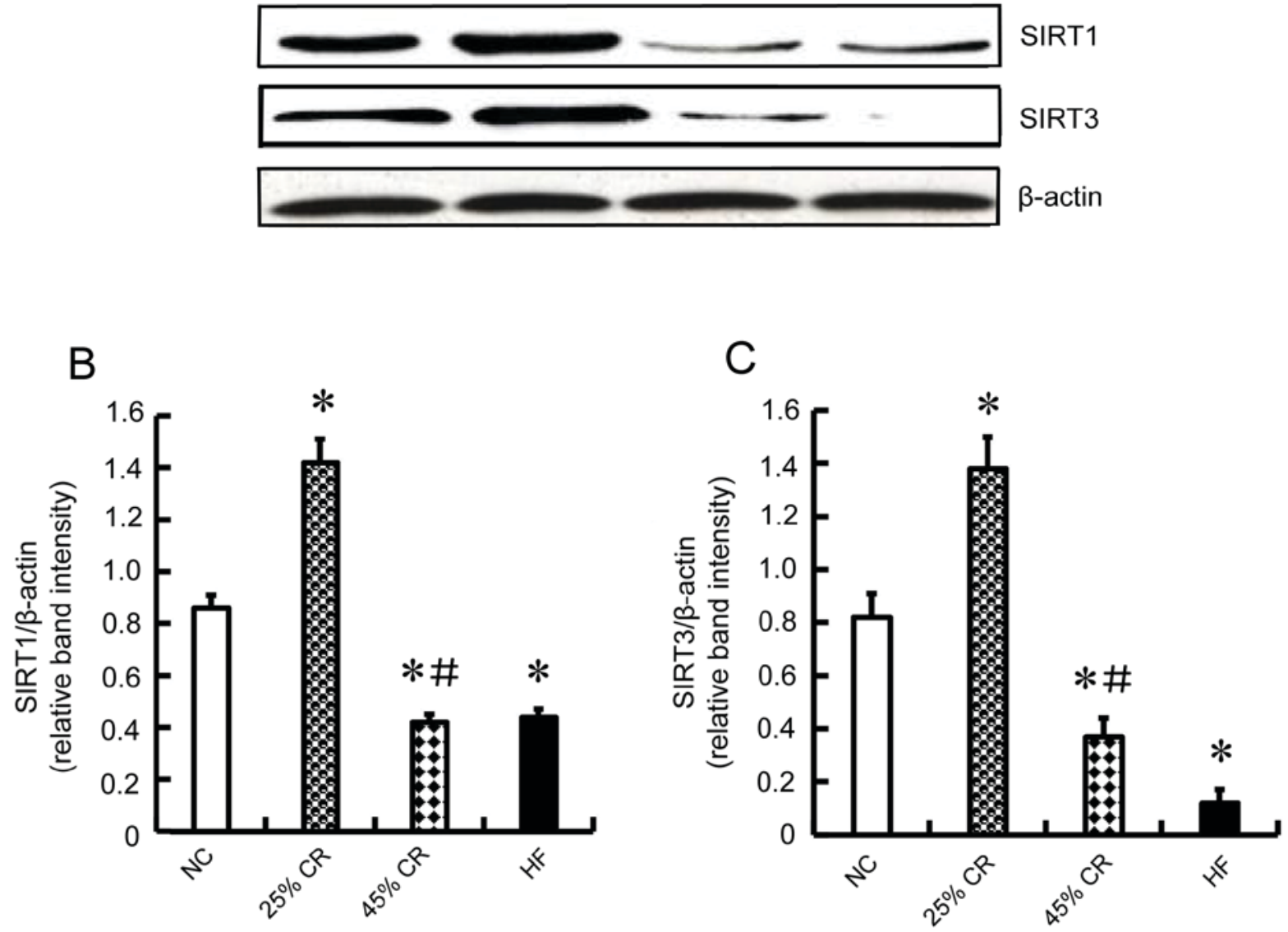

Figure 3. Effect of CR on SIRT1 and SIRT3 protein expression in the cardiac tissue. The rats were randomized into four groups (15 mice each) with similar BW, a NC group, $25,45 \% \mathrm{CR}$ and a HF group. (A) Western blot analysis revealing SIRT1, SIRT3 and $\beta$-actin protein expression in the cardiac tissue of rats. (B) SIRT1 protein abundance normalized to $\beta$-actin expression. (C) SIRT3 protein abundance normalized to $\beta$-actin expression. Data are presented as the mean \pm standard deviation. ${ }^{*} \mathrm{P}<0.05$ vs. the NC group; ${ }^{~} \mathrm{P}<0.05$ vs. the $25 \%$ CR group; $\mathrm{n}=3$. SIRT, sirtuin; $25 \% \mathrm{CR}$, a $25 \%$ calorie restriction group; $45 \% \mathrm{CR}$, $45 \%$ calorie restriction group; $\mathrm{HF}$, high-fat; $\mathrm{NC}$, negative control.

The rats HW and LVMI of the $45 \%$ CR group were the lowest. These results demonstrated that the HF group rats exhibited left ventricular hypertrophy, due to a decline of SIRT1 and SIRT3 expression. It was additionally demonstrated that the haemodynamic indices LVSP, $+\mathrm{d} P / \mathrm{d} t$ and $-\mathrm{d} P / \mathrm{d} t$ in the $45 \%$ CR group decreased, and LVEDP increased via monitoring heart function. The HF group demonstrated similar alterations, whereas the $25 \%$ CR group demonstrated an adverse alteration. It may be hypothesized and investigated in the future, that the decrease of SIRT1 and SIRT3 expression levels leads to a concomitant decline in the protective effect of cardiac tissues.

Previous studies have demonstrated that lifelong calorie restriction of $40 \%$ increases the cardiac expression of autophagic markers, which suggests that it may have a cardioprotective effect by decreasing oxidative damage brought on by aging and cardiovascular diseases $(29,30)$. In response to glucose deprivation, cardiomyocytes initiate the nuclear translocation of FoxO1 and FoxO3 to the nucleus where the transcription of genes responsible for autophagy are activated $(31,32)$. Under a starvation state, SIRT1 is upregulated $(18,33)$. SIRT1 mediates the deacetylation of FoxO1 and upregulation of Rab7, which functions as the center for mediating increased autophagic flux in response to starvation, which in turn maintains left ventricular function during these events (31). Overall, these findings unanimously support that calorie restriction may mediate its beneficial effects by stimulating autophagy in the heart, indicating the potential for cardioprotective therapy.

It is important to note that the present study had several limitations. It is well known that age-associated alterations primarily include changes in body weight, body fat, blood glucose, insulin, triglyceride and cholesterol. The present study only focused on indicators associated with cardiac function, including body weight, heart weight, left ventricle mass index, visceral fat, triglyceride and low density lipoprotein, however did not include blood glucose and insulin. The authors aim to further observe the alterations in blood glucose, insulin and other indicators in future studies. In addition, the present study only observed the effects of CR on SIRT1 and SIRT3 protein expression, but not mRNA expression. In future studies, the authors will use reverse transcription-fluorescence quantitative polymerase chain reaction to observe the mRNA expression of SIRT1 and SIRT3.

In conclusion, the data demonstrated that moderate calorie restriction $(25 \% \mathrm{CR})$, rather than severe calorie restriction (45\% CR), attenuated age-associated alterations and improved myocardial function in rats. The molecular mechanisms responsible for the protective effect of moderate calorie restriction may involve the expression of SIRT1 and SIRT3. Moderate calorie restriction may upregulate the expression 
levels of SIRT1 and SIRT3, however severe calorie restriction may lead to a decline. Therefore, the findings of the present study indicated that moderate calorie restriction may be an effective therapeutic approach for obesity-associated diseases.

\section{Acknowledgements}

Not applicable.

\section{Funding}

The present study was supported by the National Natural Science Foundation of China (grant no. 81270382/H0215).

\section{Availability of data and materials}

The datasets used and/or analyzed during the current study are available from the corresponding author on reasonable request.

\section{Authors' contributions}

WY and WW conceived and designed the study. WY and JJQ performed the experiments. WY, JJQ, CJC, YCF and WW collected and analyzed the data. WY and WW wrote the manuscript. All authors reviewed and revised the manuscript.

\section{Ethics approval and consent to participate}

The experiments were approved by the Institutional Animal Care and Use Committee of Shantou University Medical College (Shantou, China), and were conducted according to the Guide for the Care and Use of Laboratory Animals (The Regulation of Experimental Animals in Guangdong Province).

\section{Patient consent for publication}

Not applicable.

\section{Competing interests}

The authors declare that they have no competing interests.

\section{References}

1. Ginsberg HN and MacCallum PR: The obesity, metabolic syndrome, and type 2 diabetes mellitus pandemic: Part I. Increased cardiovascular disease risk and the importance of atherogenic dyslipidemia in persons with the metabolic syndrome and type 2 diabetes mellitus. J Cardiometab Syndr 4: 113-119, 2009.

2. Lavie CJ, Milani RV and Ventura HO: Obesity and cardiovascular disease: Risk factor, paradox, and impact of weight loss J Am Coll Cardiol 53: 1925-1932, 2009.

3. Civitarese AE, Carling S, Heilbronn LK, Hulver MH, Ukropcova B, Deutsch WA, Smith SR and Ravussin E; CALERIE Pennington Team: Calorie restriction increases muscle mitochondrial biogenesis in healthy humans. PLoS Med 4: e76, 2007.

4. Varady KA and Hellerstein MK: Do calorie restriction or alternate-day fasting regimens modulate adipose tissue physiology in a way that reduces chronic disease risk? Nutr Rev 66: 333-342, 2008.

5. Lefevre M, Redman LM, Heilbronn LK, Smith JV, Martin CK, Rood JC, Greenway FL, Williamson DA, Smith SR and Ravussin E; Pennington CALERIE team: Caloric restriction alone and with exercise improves CVD risk in healthy non-obese individuals. Atherosclerosis 203: 206-213, 2009.
6. Holloszy JO and Fontana L: Caloric restriction in humans. Exp Gerontol 42: 709-712, 2007.

7. Taylor DM, Maxwell MM, Luthi-Carter R and Kazantsev AG: Biological and potential therapeutic roles of sirtuin deacetylases. Cell Mol Life Sci 65: 4000-4018, 2008.

8. Al-Regaiey KA, Masternak MM, Bonkowski M, Sun L and Bartke A: Long-lived grow th hormone receptor knockout mice: Interaction of reduced insulin-like growth factor $\mathrm{i} / \mathrm{insulin}$ signaling and caloric restriction. Endocrinology 146: 851-860, 2005.

9. Fontana L, Klein S, Holloszy JO and Premachandra BN: Effect of long-term calorie restriction with adequate protein and micronutrients on thyroid hormones. J Clin Endocrinol Metab 91: 3232-3235, 2006.

10. Potente M, Ghaeni L, Baldessari D, Mostoslavsky R, Rossig L, Dequiedt F, Haendeler J, Mione M, Dejana E, Alt FW, et al: SIRT1 controls endothelial angiogenic functions during vascular growth. Genes Dev 21: 2644-2658, 2007.

11. Hsu CP, Odewale I, Alcendor RR and Sadoshima J: Sirt1 protects the heart from aging and stress. Biol Chem 389: 221-231, 2008.

12. Sundaresan NR, Gupta M, Kim G, Rajamohan SB, Isbatan A and Gupta MP: Sirt3 blocks the cardiac hypertrophic response by augmenting Foxo3a-dependent antioxidant defense mechanisms in mice. J Clin Invest 119: 2758-2771, 2009.

13. Tanno M, Sakamoto J, Miura T, Shimamoto K and Horio Y: Nucleocytoplasmic shuttling of the NAD+-dependent histone deacetylase SIRT1. J Biol Chem 282: 6823-6832, 2007.

14. Sakamoto J, Miura T, Shimamoto K and Horio Y: Predominant expression of Sir2alpha, an NAD-dependent histone deacetylase, in the embryonic mouse heart and brain. FEBS Lett 556: 281-286, 2004.

15. Sundaresan NR, Samant SA, Pillai VB, Rajamohan SB and Gupta MP: SIRT3 is a stress-responsive deacetylase in cardiomyocytes that protects cells from stress-mediated cell death by deacetylation of Ku70. Mol Cell Biol 28: 6384-6401, 2008.

16. Hafner AV, Dai J, Gomes AP, Xiao CY, Palmeira CM, Rosenzweig A and Sinclair DA: Regulation of the mPTP by SIRT3-mediated deacetylation of CypD at lysine 166 suppresses age-related cardiac hypertrophy. Aging (Albany NY) 2: 914-923, 2010.

17. Wolf G: Calorie restriction increases life span: A molecular mechanism. Nutr Rev 64: 89-92, 2006.

18. Cohen HY, Miller C, Bitterman KJ, Wall NR, Hekking B, Kessler B, Howitz KT, Gorospe M, de Cabo R and Sinclair DA: Calorie restriction promotes mammalian cell survival by inducing the SIRT1 deacetylase. Science 305: 390-392, 2004.

19. Liu L, Zhang X, Qian B, Min X, Gao X, Li C, Cheng Y and Huang J: Over-expression of heat shock protein 27 attenuates doxorubicin-induced cardiac dysfunction in mice. Eur J Heart Fail 9: 762-769, 2007.

20. Jensen MD, Ryan DH, Apovian CM, Ard JD, Comuzzie AG, Donato KA, Hu FB, Hubbard VS, Jakicic JM, Kushner RF, et al: 2013 AHA/ACC/TOS guideline for the management of overweight and obesity in adults: A report of the American College of Cardiology/American Heart Association Task Force on Practice Guidelines and The Obesity Society. Circulation 129 (25 Suppl 2): S102-S138, 2014.

21. Lee M and Aronne LJ: Weight management for type 2 diabetes mellitus: Global cardiovascular risk reduction. Am J Cardiol 99: 68B-79B, 2007.

22. Bernardi R, Cosentino ER and Borghi C: Metabolic syndrome and hypertension: Prevention and treatment. Minerva Med 97: 123-141, 2006 (In Italian).

23. Ambroskina VV, Kriachok TA, Larionov OP, Talaieva TV and Bratus' VV: Hyperlipidemia and decrease of lipid tolerance as factors of atherogenesis. Fiziol Zh 53: 19-28, 2007.

24. Li X, Zhang S, Blander G, Tse JG, Krieger M and Guarente L: SIRT1 deacetylates and positively regulates the nuclear receptor LXR. Mol Cell 28: 91-106, 2007.

25. Yu W, Zhou HF, Lin RB, Fu YC and Wang W: Short-term calorie restriction activates SIRT1-4 and -7 in cardiomyocytes in vivo and in vitro. Mol Med Rep 9: 1218-1224, 2014.

26. Alcendor RR, Gao S, Zhai P, Zablocki D, Holle E, Yu X, Tian B, Wagner T, Vatner SF and Sadoshima: Sirt1 regulates aging and resistance to oxidative stress in the heart. Circ Res 100: 1512-1521, 2007.

27. Yu W, Fu YC, Zhou XH, Chen CJ, Wang X, Lin RB and Wang W: Effects of resveratrol on $\mathrm{H}(2) \mathrm{O}(2)$-induced apoptosis and expression of SIRTs in H9c2 cells. J Cell Biochem 107: 741-747, 2009. 
28. Chen $\mathrm{CJ}, \mathrm{Fu} \mathrm{YC}, \mathrm{Yu} \mathrm{W}$ and Wang W: SIRT3 protects cardiomyocytes from oxidative stress-mediated cell death by activating NF- $\kappa$ B. Biochem Biophys Res Commun 11: 798-803, 2013.

29. Ahn J and Kim J: Nutritional status and cardiac autophagy. Diabetes Metab J 37: 30-35, 2013.

30. Wohlgemuth SE, Julian D, Akin DE, Fried J, Toscano K, Leeuwenburgh C and Dunn WA Jr: Autophagy in the heart and liver during normal aging and calorie restriction. Rejuvenation Res 10: 281-292, 2007.
31. Hariharan N, Maejima Y, Nakae J, Paik J, Depinho RA and Sadoshima J: Deacetylation of FoxO by Sirt1 plays an essential role in mediating starvation-induced autophagy in cardiac myocytes. Circ Res 107: 1470-1482, 2010.

32. Sengupta A, Molkentin JD and Yutzey KE: FoxO transcription factors promote autophagy in cardiomyocytes. J Biol Chem 284: 28319-28331, 2009.

33. Nemoto S, Fergusson MM and Finkel T: Nutrient availability regulates SIRT1 through a forkhead-dependent pathway. Science 306: 2105-2108, 2004. 